Revue des patrimoines

44 | 2021

Les patrimoines des lycées français du XIXe au XXIe siècle : de la connaissance à la valorisation

\title{
Le fonds d'archives du lycée Pierre-de-Fermat à Toulouse
}

The archival fonds of the lycée Pierre-de-Fermat, a high school in Toulouse

\section{Marilys Sanchis}

\section{(2) OpenEdition}

\section{Journals}

Édition électronique

URL : https://journals.openedition.org/insitu/30838

DOI : 10.4000/insitu.30838

ISSN : $1630-7305$

Éditeur

Ministère de la Culture

Référence électronique

Marilys Sanchis, "Le fonds d'archives du lycée Pierre-de-Fermat à Toulouse », In Situ [En ligne], 44 |

2021, mis en ligne le 30 avril 2021, consulté le 16 décembre 2022. URL : http://

journals.openedition.org/insitu/30838; DOI : https://doi.org/10.4000/insitu.30838

Ce document a été généré automatiquement le 16 décembre 2022.

\section{(c) (1) 8}

Creative Commons - Attribution - Pas d'Utilisation Commerciale - Pas de Modification 4.0 International - CC BY-NC-ND 4.0

https://creativecommons.org/licenses/by-nc-nd/4.0/ 


\section{Le fonds d'archives du lycée Pierre- de-Fermat à Toulouse}

The archival fonds of the lycée Pierre-de-Fermat, a high school in Toulouse

Marilys Sanchis

\section{Le lycée, un monument d'exception}

1 Le lycée Pierre-de-Fermat s'est développé sur le site de l'hôtel de Bernuy, construit dans la première moitié $d u \mathrm{xVI}^{\mathrm{e}}$ siècle dans le centre de Toulouse. Acheté par trois bourgeois, il fut donné à la Ville pour y fonder le 20 janvier 1567 un collège universitaire de Jésuites, qui fonctionna jusqu'en 1762. Assez vite après leur installation dans l'hôtel de Bernuy, les Jésuites agrandirent leur domaine, mais c'est surtout au XIX ${ }^{\mathrm{e}}$ siècle que l'établissement absorba beaucoup de bâtiments voisins, en particulier l'hôtel de Lestang, avant qu'il ne soit racheté par la mairie de Toulouse pour y installer le siège du rectorat de Toulouse en 1857, et le vieux couvent des Jacobins, transformé en caserne sous l'Empire. Le rôle local de l'établissement avait été suffisamment important pour justifier son maintien au moment de l'expulsion des Jésuites du royaume, en 1763. Ainsi, des lettres patentes royales du 27 novembre 1764, «portant confirmation du Collège de Toulouse", fixent les conditions de son existence comme collège royal. En 1789, il devient collège national, avant de faire place en 1795 à l'école centrale de la Haute-Garonne.

2 À la suite de la loi de 1802 établissant les lycées, et après de nombreuses difficultés matérielles, le lycée de Toulouse est véritablement fondé en 1806 par décret impérial. Dès lors, l'établissement poursuit sa vie sans interruption importante, sa dénomination changeant avec les régimes : nouveau collège royal après la Restauration en 1815 , lycée en 1848, lycée impérial en 1852, lycée de Toulouse en 1871, dénomination qu'il garde jusqu'en 1957. Il est alors baptisé lycée Pierre-de-Fermat, en hommage au grand mathématicien du XVII ${ }^{e}$ siècle né à Beaumont-de-Lomagne et conseiller au parlement de Toulouse. $\mathrm{Au}$ cours des $\mathrm{XIX}^{\mathrm{e}}$ et $\mathrm{Xx}^{\mathrm{e}}$ siècles, le lycée voit la création de classes préparatoires aux grandes écoles (Polytechnique, Saint-Cyr, l'École navale, l'École 
centrale des arts et manufactures, l'École normale supérieure, l'École des mines, l'Institut agronomique, l'École des chartes...). Développées au $\mathrm{xx}^{\mathrm{e}}$ siècle, ces dernières confèrent à l'établissement un prestige qui l'élève au rang des meilleurs lycées de France. Son rayonnement et sa réputation d'excellence attirent un nombre important d'étudiants originaires de toutes les régions de France et de l'étranger.

L'histoire du lycée est indissociable de l'Association des anciens de Fermat. Créée en 1872, l'Association des anciens élèves du lycée de Toulouse, Association des anciens élèves du lycée Pierre-de-Fermat depuis 1957, a pour objectif premier de «conserver, resserrer ou établir des relations amicales entre les anciens ", en étroite collaboration avec les différentes administrations de l'établissement. Un fonds d'archives composé principalement de photographies relatives à la vie de cette association est conservé aux archives départementales de la Haute-Garonne ${ }^{1}$.

\section{Patrimoine immobilier et mobilier}

4 L'hôtel de Bernuy, proche du cloître et de l'église des Jacobins, est l'un des plus beaux hôtels particuliers de la ville de Toulouse, construit grâce à la prospérité apportée par le commerce du pastel au XvI ${ }^{\mathrm{e}}$ siècle. La qualité et l'inventivité du travail des architectes et des sculpteurs qui ont participé à son édification en font un chef-d'œuvre de l'architecture Renaissance à Toulouse. Le richissime commerçant pastelier Jean Bernuy, qui devint capitoul en 1533, fit ériger cet hôtel par Louis Privat dans le premier tiers du $\mathrm{XVI}^{\mathrm{e}}$ siècle. Construit dans le style de la première Renaissance, sa tour d'escalier hexagonale est l'une des plus hautes de la ville. En 1605, les capitouls font élever le grand portail du collège, orné de leurs armes, de celles du roi et du monogramme de Jésus IHS, qui ouvre sur la rue des Jacobins, aussi appelée rue des Jésuites (actuelle rue Lakanal). Ce portail monumental ${ }^{2}$, où s'affiche le mécénat des capitouls, est protégé au titre des monuments historiques depuis 2007 [fig. 1], tandis que l'hôtel lui-même a été classé monument historique en $1889^{3}$. Au titre de sa compétence sur les collèges, le conseil départemental de Haute-Garonne a entrepris en 2019, en étroite collaboration avec les services de la Drac Occitanie, la restauration de ce portail ainsi que celle de la porte d'entrée du lycée qui date du début du XVI ${ }^{e}$ siècle ; les travaux sont actuellement en cours d'achèvement. Il est prévu de les poursuivre en 2021, sur les deux cours du collège, elles aussi classées. 
Figure 1

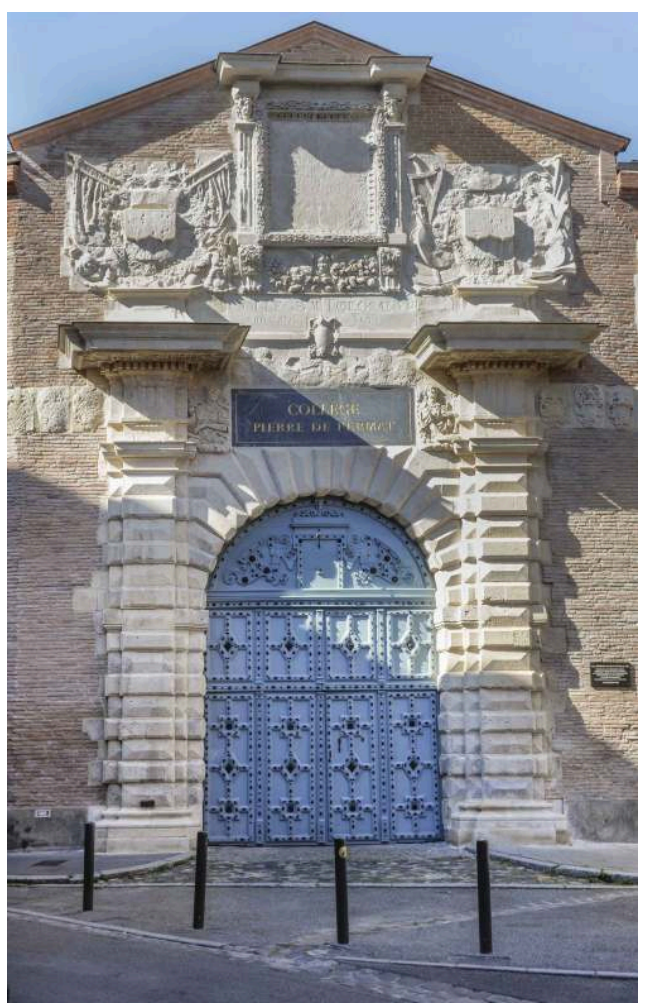

Portail de l'ancien collège des Jésuites de Toulouse (1605), actuel collège Pierre-de-Fermat, 2020.

(c) Bruno Venzac (Conseil départemental de la Haute-Garonne, direction des Archives et du Patrimoine culturel).

5 En ce qui concerne le patrimoine mobilier remarquable du lycée, on trouve dans les recensements réalisés par le service de l'Inventaire général de la région Occitanie le buste en bronze du mathématicien Pierre de Fermat ${ }^{4}$, sculpté en 1968 par Marcel Chauvenet (1906-1988), placé dans le hall d'entrée du lycée. Le monument commémoratif aux morts de la guerre 1914-1918 du lycée figure également sur l'inventaire ${ }^{5}$; il a été érigé à l'initiative de l'Association des anciens élèves du lycée, conçu en marbre blanc par l'architecte Paul Bonamy et le marbrier Bernard Doat, et remis solennellement au proviseur du lycée par le président de l'Association, César Bru, en 1921.

\section{Genèse d'une collecte}

\section{Caractère patrimonial du fonds d'archives}

6 Compte tenu du prestige et de l'ancienneté de ce lycée toulousain, ses archives présentent un intérêt historique et patrimonial évident : elles offrent aux chercheurs une vision globale de l'évolution du système éducatif et du cursus des élèves du lycée, depuis leur inscription jusqu'à leur entrée en classes préparatoires aux concours d'accès aux grandes « Écoles du Gouvernement » fondées par Napoléon.

7 Les pistes à explorer sont nombreuses, non seulement pour une étude monographique de l'établissement mais aussi pour l'histoire de l'enseignement: démocratisation de l'enseignement aux $\mathrm{XIX}^{\mathrm{e}}$ et $\mathrm{XX}^{\mathrm{e}}$ siècles, scolarisation de masse qui s'amplifie au cours 
du XXe siècle, analyse de la réussite ou de l'échec des réformes successives de l'enseignement secondaire, variations des contenus pédagogiques, scolarisation progressive des jeunes filles, évolution de la population scolaire en termes de fréquentation et de statistiques, dans un lycée autrefois réservé aux fils de la bourgeoisie.

Le lycée ne peut qu'avoir intérêt à confier son fonds aux archives départementales (audelà de l'obligation légale) : son patrimoine historique est préservé en un lieu unique dédié à sa bonne conservation et les relations tissées avec l'institution départementale servent les intérêts des professeurs d'histoire chargés d'initier leurs élèves des classes préparatoires aux carrières en lien avec les archives.

\section{Règlementation}

9 Les archives départementales ont pour mission de contrôler les conditions de conservation et d'élimination des archives publiques constituées dans le périmètre du département, mais ont aussi pour rôle d'aider les services à normaliser et à fluidifier leur production documentaire, en les accompagnant notamment dans la mise en place des procédures et des outils de circulation de l'information. Elles visent leurs demandes de versement et d'élimination dans les meilleurs délais. Enfin, les archives départementales collectent les documents présentant un intérêt historique dès lors que les durées de conservation légales sont échues ; elles en assurent leur classement et en produisent des inventaires, indispensables à la communication. Elles assurent la bonne conservation des fonds qui leur sont versés : accès contrôlés à leurs locaux, protection contre les risques d'effraction et d'incendie, entretien régulier des magasins d'archives, veille et migration périodique pour les archives électroniques. Elles veillent également à communiquer les archives au public conformément aux dispositions législatives en vigueur.

De manière générale, la collecte des archives des établissements scolaires a été tardive, à l'image de celle des administrations ou des collectivités territoriales en général. En effet, la première instruction de la direction des Archives de France qui règle le sort des archives des établissements d'enseignement date du 26 mai $1970^{6}$; elle impose sur le plan national à tous les établissements scolaires de verser en priorité les archives antérieures à 1940. En revanche, les archives postérieures à 1940 ne seront versées que par quelques établissements sélectionnés : un échantillon représentatif d'un dixième des établissements de la Haute-Garonne est alors constitué et quelques établissements, dont le lycée Fermat, sont désignés pour verser leurs archives. Les lacunes constatées aux archives départementales de Haute-Garonne dans les fonds d'archives des établissements scolaires résultent de cette méthode de sélection. En effet, à ce jour, quatorze collèges et neuf lycées seulement y ont versé leurs archives. Les versements en provenance du lycée Pierre-de-Fermat effectués à la suite de cette instruction se répartissent en plusieurs lots: le premier en 1971, un deuxième en 1979, puis un troisième en $1983^{7}$. Ces versements viennent compléter les fonds d'archives modernes (entre 1800 et 1940) produits par la préfecture de région et classés en série $\mathrm{T}$ aux archives départementales.

11 En raison de l'évolution législative, réglementaire et administrative mais aussi de l'augmentation du nombre des élèves, et au vu d'un accroissement incontestable de la masse des documents produits par les services et établissements concourant à 
l'éducation nationale, une refonte des dispositions relatives au traitement des archives a été opérée pour aboutir à une nouvelle circulaire, en date du 22 février $2005^{8}$. Celle-ci a annulé les dispositions de l'instruction de 1970. Aboutissement d'une réflexion sur l'évaluation, la sélection et la collecte des archives de l'Éducation nationale, elle vise aussi à assurer dans les meilleures conditions la conservation des archives définitives sur lesquelles se fonde la recherche historique et scientifique. Dans une approche thématique, elle présente pour chaque secteur d'activité des tableaux de tri et de conservation, ou tableaux d'archivage, dont l'objectif est de faciliter, conformément à la circulaire du Premier ministre du 2 novembre $2001^{9}$, une bonne gestion des archives courantes et intermédiaires de la part des services producteurs des documents, ainsi qu'une collecte sélective et raisonnée des archives historiques à l'expiration des délais d'utilité administrative ${ }^{10}$.

Les relations entre les archives départementales et les établissements scolaires de la Haute-Garonne sont restées occasionnelles depuis la première circulaire de 1970: un établissement d'enseignement primaire ou secondaire, dépourvu d'archiviste, où la gestion des archives est souvent confiée à un documentaliste ou un intendant, sollicite les archives départementales dès lors que le local d'archives est saturé ou par exemple, pour réguler le flux de copies d'examens lorsque le collège ou le lycée a été centre d'examen du brevet ou du baccalauréat. Les fonds relatifs à l'enseignement primaire et secondaire conservés aux archives départementales, résultant de ces relations épisodiques, représentent environ 180 mètres linéaires pour une période allant de 1800 à aujourd'hui. En l'absence de collecte volontariste et pour remettre la gestion des archives au cœur des préoccupations d'un établissement scolaire, les archives départementales de Haute-Garonne ont souhaité aller au-devant des attentes et mettre en avant leur responsabilité de conseil et de contrôle. C'est pourquoi, après la parution de l'instruction de 2005 et en vue de sensibiliser les établissements à la pratique archivistique de manière concrète, les archives départementales ont rédigé un guide d'archivage à l'usage des collèges et lycées, conçu de manière didactique pour les gestionnaires d'archives en établissements scolaires qui n'ont pas de formation archivistique. Ce guide reprend les grandes thématiques abordées dans l'instruction, sous forme de tableaux d'archivage simplifiés. Il apporte aide et conseil aux collèges et lycées pour toutes les questions liées à la gestion quotidienne de leurs documents : classement des dossiers courants, aménagement de salle d'archives, tris, élimination, versement, etc. Sa diffusion a été assurée auprès de tous les collèges de la HauteGaronne par le conseil départemental et pour les lycées de l'académie de Toulouse, par le biais du rectorat. Sa version électronique est disponible sur le site internet des archives départementales de la Haute-Garonne ${ }^{11}$. On note en page de garde le choix de l'illustration de ce guide : la porte de l'hôtel de Bernuy.

\section{Méthodologie}

13 Les archives départementales ont été contactées par l'intendant du lycée Fermat en 2012, lors de travaux engagés sur les bâtiments du lycée. Ces travaux avaient entraîné l'externalisation de la conservation des archives chez un prestataire agréé de la périphérie toulousaine, opération pilotée et financée par les services de la région, avec l'accord des archives départementales. L'externalisation d'archives publiques est une procédure encadrée par le Code du patrimoine qui consiste à effectuer un transfert 
physique d'archives courantes et intermédiaires d'une collectivité vers un lieu de stockage privé géré par un prestataire agréé par les Archives de France.

Les archives départementales ont conduit une inspection, très bien accueillie par l'établissement, soucieux de recevoir avis et conseils. Une visite du site d'externalisation des archives du lycée a ainsi été effectuée : 300 à 400 mètres linéaires d'archives conditionnées en cartons sommairement identifiés étaient stockés sur des palettes dans un local adapté, en attente de traitement. Une réflexion s'est engagée sur les méthodes appropriées au rapatriement et au classement de ces arriérés d'archives dans les nouveaux locaux du lycée. La question de la conservation des archives de cet établissement prestigieux, qui ne disposait pas d'archiviste à demeure et qui ne souhaitait pas saturer d'emblée ses nouveaux locaux d'archives, s'est immédiatement posée.

15 Les archives départementales n'ont pas pour vocation ni politique de se substituer aux services pour le traitement de leurs archives. Toutefois, une décision au caractère exceptionnel a été prise: de 2017 à 2019, les archives ont été rapatriées du site d'externalisation vers le lycée ; à raison d'une demi-journée par semaine, un agent des archives départementales s'est consacré au tri des archives, plus ou moins en vrac, dans un local du lycée dédié à cette opération de longue haleine. Une telle intervention, ciblée et rarissime, dans ce type de structure, tient au caractère prestigieux du lycée et à la nature de son fonds, et au fait que la collecte d'archives d'établissements scolaires ait été tardive sur le territoire.

16 Le traitement engagé sur le long terme dans les locaux du lycée s'est traduit par la réception de lots de cartons d'archives, ramenés depuis le site d'externalisation au fur et à mesure et de façon aléatoire, dont le contenu a été soigneusement étudié et la destination déterminée en application de la règlementation en vigueur ${ }^{12}$. Des archives dont le sort final ${ }^{13}$ était la destruction ont été recensées sur des bordereaux d'élimination soumis au visa de la directrice des archives départementales avant leur destruction matérielle ${ }^{14}$. Les archives vouées à une conservation définitive ont été regroupées sur place, classées selon un cadre logique établi au vu des typologies présentes et inspiré de l'instruction de 2005, conditionnées en boîtes d'archives classiques ${ }^{15}$ mises à disposition par le lycée, puis préparées en vue de leur versement aux archives départementales. Les archives intermédiaires dont la durée d'utilité administrative n'était pas échue et qui devaient donc être conservées par l'établissement ont été identifiées pour être regroupées de façon rationnelle par service producteur ${ }^{16}$ et stockées dans les nouveaux locaux d'archives du lycée. Deux à trois agents du lycée ont été mis à contribution une demi-journée par semaine pour rassembler, classer sommairement les dossiers d'élèves dans l'ordre alphabétique, les mettre en boîtes d'archives puis saisir sur un fichier informatique les noms des premiers et derniers dossiers contenus dans chaque boîte, fichier transmis ensuite aux archives départementales et utilisé pour rédiger le bordereau de versement.

17 Le bordereau de versement officialisant la prise en charge des archives du lycée par les archives départementales a été rédigé en fonction de l'avancée des opérations de tri et de classement. Des transferts réguliers aux archives départementales ont dû être opérés au fur et à mesure pour laisser la place au lot suivant de cartons à traiter dans le local du lycée. De cette "noria" (prestataire d'archivage agréé - lycée - archives départementales) ont résulté cinq versements successifs ${ }^{17}$, chacun étant organisé selon le même plan de classement thématique prédéfini, tous regroupés dans un bordereau 
de versement méthodique unique. Lorsque le bordereau de versement a été achevé, une mise à jour du répertoire méthodique "Éducation- Enseignement primaire et secondaire ", mis à la disposition du public en salle de lecture et sur le site internet des archives départementales ${ }^{18}$, a été réalisée. On y trouve le classement thématique de tous les fonds des établissements scolaires de la Haute-Garonne qui ont versé leurs archives, par niveau d'enseignement : écoles / collèges / lycées.

Les archives départementales ont engagé une réflexion sur le sort final qui serait réservé aux dossiers d'élèves. L'instruction de 2005 prône un échantillonnage alphabétique ou chronologique, avec soit une conservation des dossiers individuels dont les patronymes commencent par les lettres $\mathrm{B}$ et $\mathrm{T}$, soit une conservation des dossiers pour les années scolaires se terminant en 0 et 5 . Ce type d'échantillonnage ne tient pas compte du volume initial de l'ensemble de documents et n'a pas de réelle valeur statistique, dans la mesure où il omet des pans entiers de la population. Le cadre méthodologique pour l'évaluation, la sélection et l'échantillonnage des archives publiques de 2014 a rationnalisé les critères d'échantillonnage des archives publiques ${ }^{19}$ : avant toute décision de sélection d'une série de dossiers à conserver, il convient de procéder à une évaluation, c'est-à-dire une opération archivistique visant à déterminer l'intérêt public, administratif, juridique ou historique de l'ensemble de documents considéré. La décision d'échantillonner un fonds écarte de fait les possibilités ultérieures de recherche nominative individuelle, qu'elle ait un caractère administratif ou généalogique. Au terme d'une réflexion prenant en compte l'ensemble de ces éléments, les archives départementales de Haute-Garonne ont choisi de conserver intégralement les dossiers d'élèves, soit environ 30 mètres linéaires, chacun d'entre eux présentant un intérêt intrinsèque sur le plan de l'histoire locale, personnelle et familiale.

19 À la fin de ces opérations, la collecte représentait plus de $500 \operatorname{articles}^{20}$, soit plus de 56 mètres linéaires; plus de 100 mètres linéaires de documentation nationale, de dossiers de gestion comptable, d'états récapitulatifs d'heures supplémentaires, de demandes d'inscription d'élèves restées sans suite, de correspondance relative aux fonds sociaux, d'instructions sur le déroulement du baccalauréat ou de certificats médicaux ont été éliminés par les soins du service logistique du lycée au fur et à mesure de l'avancée des opérations de tri. Cinq versements successifs ont résulté de cette intervention, étalés sur deuxans, avec un seul numéro d'entrée aux archives départementales attribué pour plus de cohérence ${ }^{21}$. L'ensemble des archives collectées était en bon état; tout a été coté et reconditionné en boîtes de conservation sur mesure.

La collaboration entre le lycée et les archives départementales est désormais engagée sur le long terme, le lycée ayant décidé de verser chaque année sa production la plus volumineuse, c'est-à-dire les dossiers d'élèves dont la durée d'utilité administrative de cinquante ans est échue : début 2020, le versement des dossiers des élèves sortis du lycée en 1969 a été effectué. Les archives départementales veilleront sur le sort des autres typologies documentaires, qui seront traitées sur le long terme de la même façon: en accord avec la direction du lycée, les éliminations réglementaires seront régulièrement pratiquées par les agents du lycée et d'autres versements seront organisés. 


\section{Classement}

\section{Typologies documentaires rencontrées}

21 Les dossiers d'élèves sortis du lycée entre 1958 et 1969 représentent une partie volumineuse du fonds collecté2 ${ }^{22}: 308$ dossiers, soit 30 mètres linéaires. Ils prennent la suite des répertoires alphabétiques et livres-journaux des entrées et sorties des élèves (1886-1958), fiches et notices individuelles d'inscription des élèves (1895-1960) présents dans les versements précédents aux archives départementales. La description des dossiers a été complétée aux archives départementales par une indexation fine aux nom et prénom des élèves pour faciliter la recherche. On trouve également dans ce versement les fiches individuelles de renseignements ainsi qu'un fichier récapitulatif des personnels administratifs et enseignants nés entre 1890 et 1943, répertoriés par patronyme, classés dans l'ordre alphabétique ${ }^{23}$. Ces documents viennent compléter les collections de dossiers individuels administratifs des personnels du lycée nés entre 1889 et 1919 déjà conservées aux archives départementales ${ }^{24}$.

Un sous-fonds a été constitué pour la collection des bulletins d'information ALTIER («Anciens du lycée de Toulouse internes et externes réunis») de 1957 à $2004^{25}$. Ce bulletin, d'abord annuel puis trimestriel, témoin de l'Association des anciens de Fermat, retrace la vie du lycée à travers les contributions des anciens qui font vivre l'association.

On trouve également dans le versement un registre de procès-verbaux des séances du conseil de professeurs entre 1911 et $1916^{26}$, ainsi que des enquêtes statistiques sur la situation et la scolarité des élèves entre 1959 et $1994^{27}$.

À l'occasion de la commémoration du bicentenaire du lycée, en 2006, des documents considérés comme remarquables pour l'histoire de l'établissement avaient été regroupés par les personnels du lycée afin d'être présentés dans une exposition. Cette sélection de documents, composée en partie d'originaux, a été retrouvée lors de notre campagne de collecte, puis classée et intégrée au versement. Elle permet d'entrevoir la construction de la mémoire du lycée autour de quelques thématiques récurrentes. $\mathrm{Si}$, bien évidemment, figurent le règlement intérieur du lycée avec prix de pension et trousseau (1855) et le rapport de présentation du lycée impérial ${ }^{28}$ (1864) [fig. 2], les bâtiments se taillent la part du lion: originaux et copies numériques de plans des bâtiments du lycée entre 1872 et 1896 ; dossier du projet de reconstruction du lycée avec plans et coupes, travaux d'aménagement à l'hôtel de Bernuy (1861); extrait de procès-verbal du conseil d'administration de l'Association des anciens élèves avec description des locaux ${ }^{29}$ (1942); baux à ferme avec état des lieux de la ferme du domaine de Bellevue, où fut implantée l'annexe du lycée Fermat, ainsi qu'actes et contrats concernant le terrain ${ }^{30}$ (1896-1940). Naturellement, les élèves ont aussi la part belle: archives sur leur vie quotidienne au fil du temps, avec la photographie d'une chambre d'internat; menus $(1939-1941,1969,1980)$; composition du trousseau réglementaire $^{31}$ (1937) [fig. 3]; reproduction des bulletins trimestriels de Georges Pompidou, élève de la classe d'hypokhâgne du lycée Fermat en 1928-1929 [fig. 4]; rapports d'incidents lors des événements de Mai $1968^{32}$; cahiers de palmarès et de distribution solennelle des prix aux élèves du lycée entre 1921 et $1968^{33}$. Enfin, dans cette sélection de documents des $\mathrm{XIX}^{\mathrm{e}}$ et $\mathrm{xx}^{\mathrm{e}}$ siècles on trouve le dossier préparatoire et 
la version finale du livre du bicentenaire Le Lycée Pierre-de-Fermat, 1806-2006 édité pour la circonstance par l'Association des anciens élèves de l'établissement ${ }^{34}$.

\section{Figure 2}

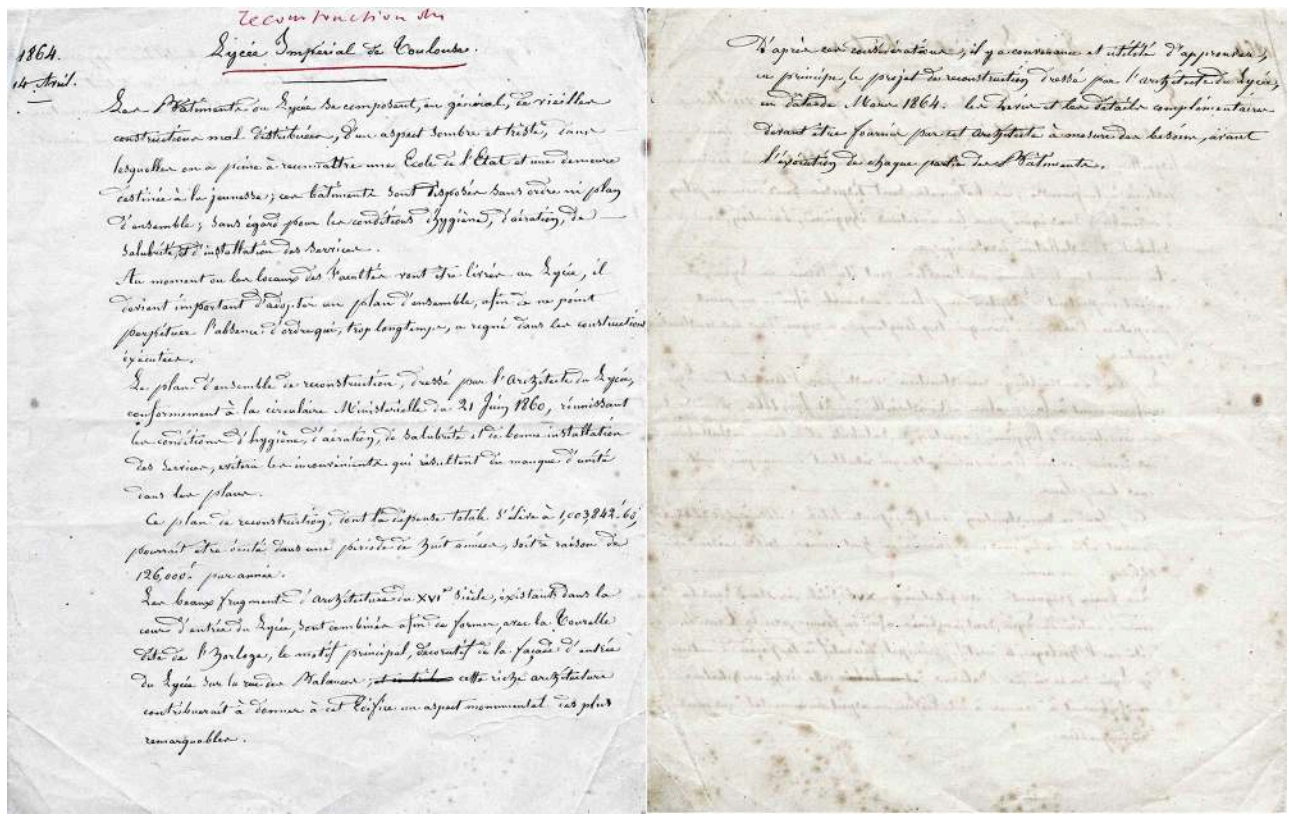

Rapport de présentation du lycée impérial de Toulouse approuvant le plan de reconstruction des bâtiments dressé par l'architecte du lycée sur une période de huit ans (14 avril 1864). Versement du lycée Pierre-de-Fermat aux archives départementales de la Haute-Garonne (8090 W 360).

Reproduction Bruno Venzac (Conseil départemental de la Haute-Garonne, direction des Archives et du Patrimoine culturel). 
Figure 3

Les frais d'études et de pension sont payables sans avertissement en
ois parties égales : au début d'octobre, de janvier et d'avril. Les chèquee trois parties égales : au début d'octobre, de jan
doivent être payables sans frais à Toulouse. Tout élève ancien qui rentre avant le $1^{\text {or }}$ novembre doit le premier

Tous les autres frais scolaires sont dus à partir du $\mathrm{I}^{\text {er }}$ ou du 16. Tout trimestre commencé est dû en entier.

En ce qui concerne les élèves algériens, tunisiens, des colonies françaises ou etrangers, les frais solaires doivent etre régles a la la caiss

TROUSSEAU

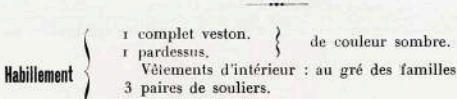
2 paires de gants.

Io chemises de jour (qui ne déteignent pas au blanchis-

sage).
chemises de nuit.

8 paires de chunt.

18 mouchoirs.

8 caleçons (obligatoires).

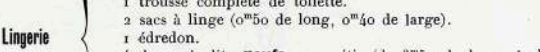
4 draps de lits neuts en métis (de $3^{\mathrm{m}} \mathrm{m}_{5}$ de long et $2^{\mathrm{m}}$ Araps de lits neurs ch te
$\mathrm{a}^{2} 2^{\mathrm{m}_{10}}$ de large). 8 serviettes neuves

$\left.\mathrm{o}^{\mathrm{m}} 75\right)$ et 6 mains-épong

作elopes blanches pour serviettes de table.

(Ges dimensions seront rigoureusement exigées.)
maillots blancs et deux culottes blanches pour la gyn

nastique.

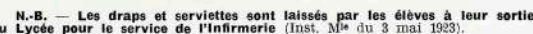
Pour les eleves qui suivent les cours des Grandes Ecoles, et qui ne
peuvent pas apporter leur trousseau d'un autre Lycé ou College, les fa-
milles peuvent se dispenser de fournitu les draps et les serviettes moyen. nant un abonnement ainuel de 117 francs pour les pensionnaires, 36 francs pour les demi-pensionnaires. Les familles doivent se pourvoir elles-mémes de tous les objels com
Le moyen de payement le plus pratique est le chèque postal (M. I' $\mathrm{Eco}$

L'uniforme n'est plus obligatoin'.

Le blanchissage du linge et le menu raccommodage des vêtements

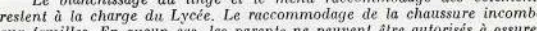
eux-mines le blanchiseage dus linge de leurs enfants Les demi-pensionnaires doivent fournir six serviettes de table qui teront pour le service de l'Infirmerie au départ de ces éleves. Tous les objets de lingerie et de vestiaire doivent être marqués au
méro de l'eléve à laide de numéros brodés en rouge sur fond blanc e numéro de l'éléve
ne déteignant pas.

Le numéro est indiqué, sur demande, par ladministration du Lycée.

En ce qui concerne les vätements, les chaussures, les malles et les valises
objets etant a la disposition des edeves qui les emportent librement tors

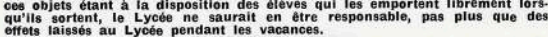

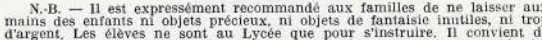

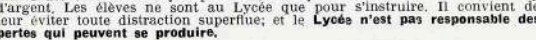

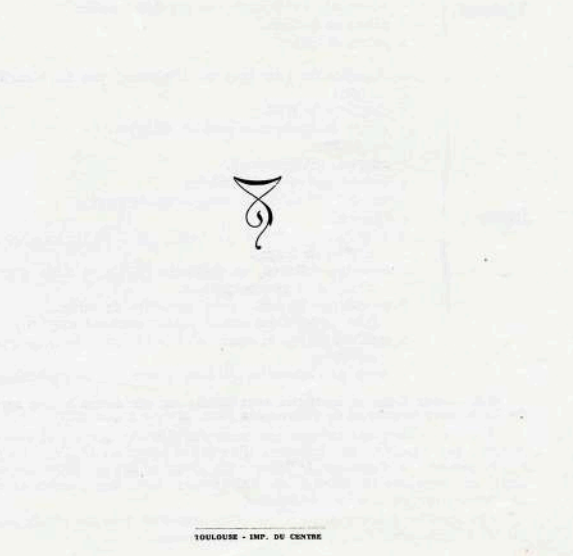

Composition du trousseau réglementaire pour les élèves internes au lycée de garçons de Toulouse où l'uniforme n'est plus obligatoire depuis la Première Guerre mondiale, extrait du « Fascicule d'admission avec tarifs de la rétribution scolaire » (1937). Versement du lycée Pierre-de-Fermat aux archives départementales de la Haute-Garonne (8090 W 362).

Reproduction Bruno Venzac (Conseil départemental de la Haute-Garonne, direction des Archives et du Patrimoine culturel) 
Figure 4

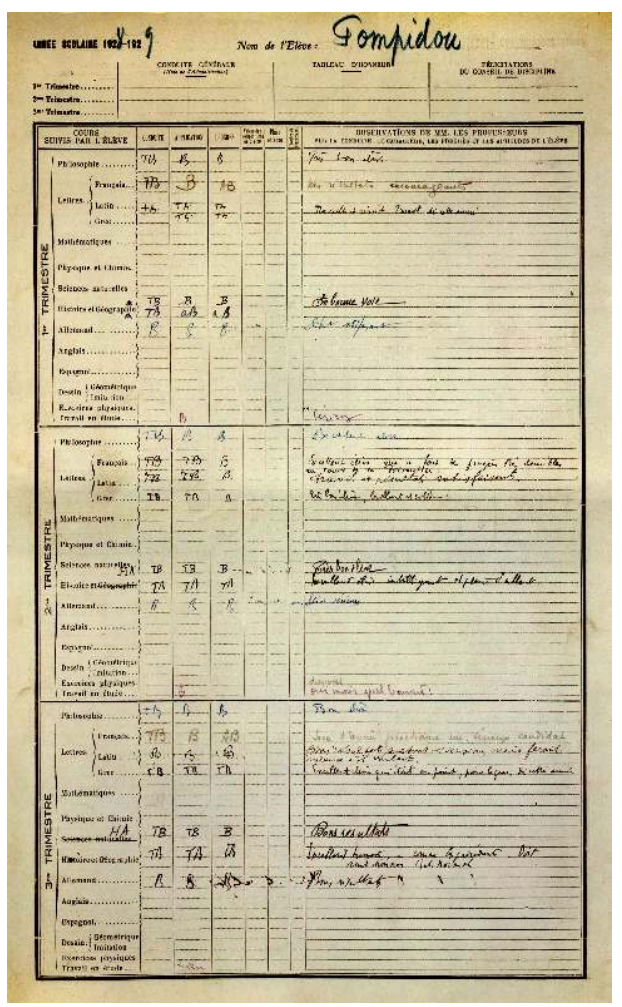

Un des bulletins trimestriels de Georges Pompidou, élève en classe d'hypokhâgne au lycée Fermat (1928-1929). Versement du lycée Pierre-de-Fermat aux archives départementales de la HauteGaronne (8090 W 362).

Reproduction Bruno Venzac (Conseil départemental de la Haute-Garonne, direction des Archives et du Patrimoine culturel).

L'opération de traitement des archives du lycée a également permis de collecter une autre typologie : des collections originales de photographies de classe et du personnel enseignant de 1871 [fig.5], puis de 1903 à 2011, en partie identifiées, classées chronologiquement par année scolaire et par classe ${ }^{35}[\mathrm{fig} .6]$; on trouve aussi une reproduction de la photographie de la première équipe de rugby de la société sportive du lycée nommée La Violette de Toulouse ${ }^{36}$ (1894) [fig. 7]. 
Figure 5

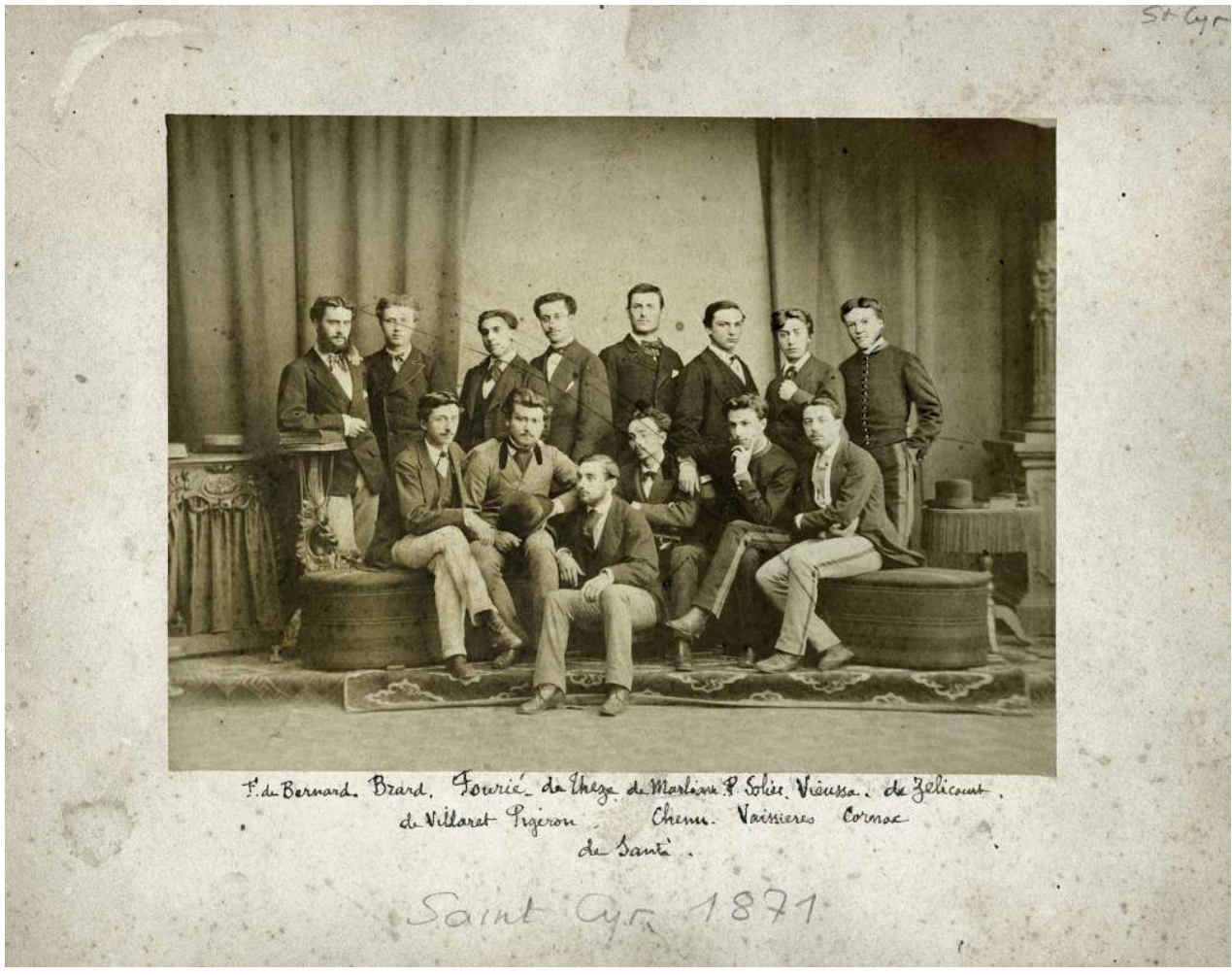

Classe préparatoire de Saint-Cyr du lycée de Toulouse (1871). Versement du lycée Pierre-de-Fermat aux archives départementales de la Haute-Garonne (8090 W 48).

Reproduction Bruno Venzac (Conseil départemental de la Haute-Garonne, direction des Archives et du Patrimoine culturel) 
Figure 6

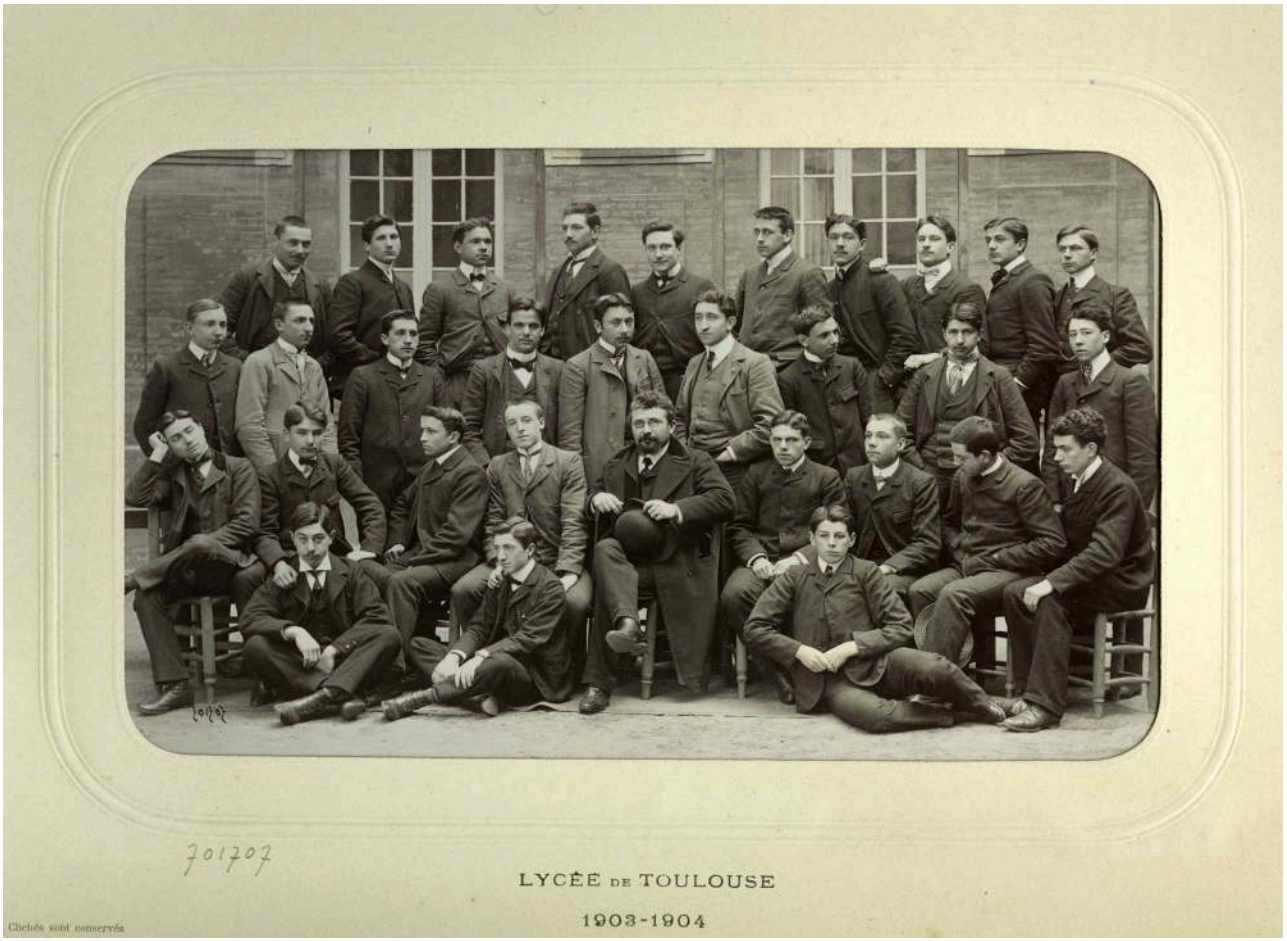

Classe de rhétorique du lycée de Toulouse (1903-1904). Versement du lycée Pierre-de-Fermat aux archives départementales de la Haute-Garonne (8090 W 48).

Reproduction Bruno Venzac (Conseil départemental de la Haute-Garonne, direction des Archives et du Patrimoine culturel). 


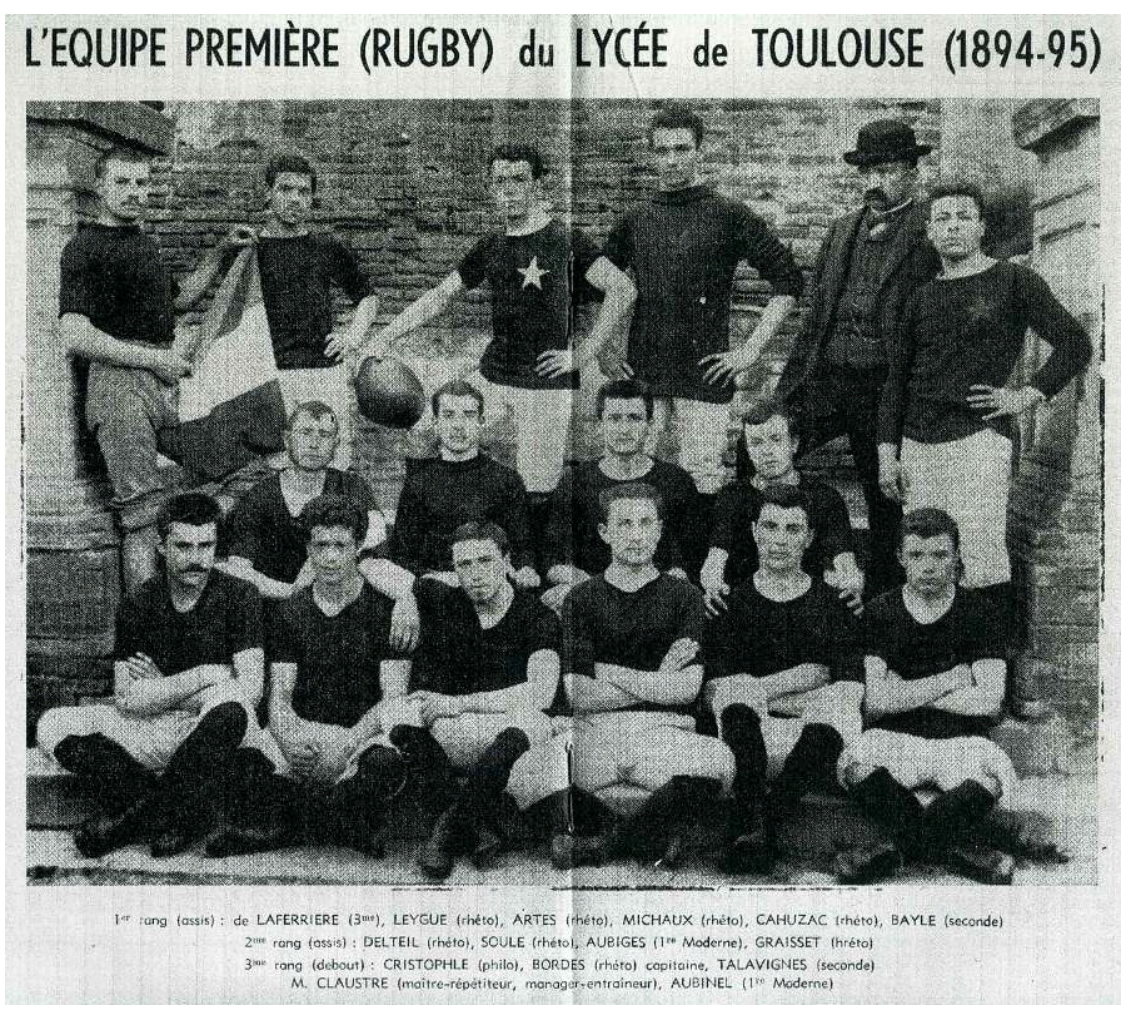

Coupure de presse montrant l'équipe de rugby de la société sportive du lycée nommée La Violette de Toulouse (1894). Versement du lycée Pierre-de-Fermat aux archives départementales de la HauteGaronne (8090 W 332).

Reproduction Bruno Venzac (Conseil départemental de la Haute-Garonne, direction des Archives et du Patrimoine culturel).

\section{Après la collecte et le classement : la communication et la valorisation}

La communication et la valorisation de ce fonds ont été l'aboutissement naturel de la chaîne du traitement archivistique après deux années de collecte et de tri.

Les conditions d'accès à ces archives sont fixées suivant les délais de communicabilité prévus par le Code du patrimoine (art. L213-2). Les dossiers scolaires des élèves sont ainsi couverts par un délai de communicabilité de 50 ans, en vertu du respect de la vie privée, mais aussi parce qu'ils peuvent comporter des jugements de valeur ou appréciations sur une personne physique. Les documents administratifs tels que les registres de procès-verbaux de séances de conseils d'administration et des commissions, ou tout dossier concernant la vie de l'établissement tel que des rapports, des circulaires ou de la correspondance sont communicables immédiatement. Les registres d'infirmerie du lycée ${ }^{37}$ ainsi que les fiches individuelles de consultation en infirmerie sont soumis à un délai de communicabilité de 120 ans après la naissance de la personne concernée ou bien 25 ans après son décès, au titre de la protection du secret médical.

28 Le fonds est mis à la disposition du public dans la salle de lecture des archives départementales à des fins de consultation par des chercheurs qui travaillent sur 
l'histoire des établissements scolaires, l'histoire sociale, l'histoire de l'éducation ou encore sur un personnage illustre ayant été scolarisé dans ce lycée. De même, le fonds se prête à la recherche généalogique. Pour exemple, un chercheur a contacté les archives départementales car il souhaitait retrouver une photographie de son ancêtre jeune. Il n'avait pour point de départ que sa date de naissance, mais supposait qu'il avait été élève au lycée Fermat. La recherche a été orientée vers les registres d'inscription et les listes d'élèves. Après avoir trouvé son aïeul, identifié les dates de sa scolarisation et les classes fréquentées, le chercheur a pu consulter les photographies de classe correspondantes; il a ainsi retrouvé la photographie de son ancêtre à l'époque où il était élève à Fermat, qu'il a facilement reconnu parmi tous les autres élèves.

Deux exemples de communication et de recherches abouties démontrent l'intérêt d'une indexation nominative fine des dossiers individuels. Le premier concerne un ancien élève du lycée Fermat, Bernard Maris (1946-2015), professeur des universités, journaliste, économiste, tué lors de l'attentat contre Charlie Hebdo à Paris, en janvier 2015. En janvier 2020, le lycée a voulu donner son nom à un nouveau bâtiment, l'un des plus grands de l'établissement. Son dossier scolaire (1953-1963) a été rapidement repéré dans la base de données des archives départementales et ainsi aisément communiqué ${ }^{38}$. On y trouve les bulletins de notes de ses années de lycée, des notes concernant son orientation, des fiches de renseignements et d'état civil, de la correspondance, des certificats. Ainsi a été retracé le parcours de Bernard Maris, qui a fait toute sa scolarité au lycée de 1956 à 1963, y a été surveillant de 1964 à 1967 puis parent d'élève. Il a marqué l'établissement de son empreinte; une plaque à son nom a été apposée sur l'un des murs du bâtiment inauguré.

Un deuxième exemple concerne Roger Fontaneau (1919-1968), ancien professeur d'histoire au lycée Fermat de 1944 à 1967, chevalier des palmes académiques en 1961. Des renseignements sur sa carrière de professeur ont été demandés par la direction du lycée qui souhaitait apposer sur les murs du lycée une plaque en sa mémoire à l'occasion de son décès, en septembre 2019, car il a été honoré du titre de «Juste parmi les nations » avec son épouse Raymonde. Une recherche par patronyme sur la base de données des archives départementales a là aussi permis de localiser rapidement la fiche individuelle de carrière de ce professeur et de communiquer au lycée les éléments demandés ${ }^{39}$.

\section{Opérations ponctuelles}

31 Lors de la collecte des archives du lycée Fermat, un document remarquable a été particulièrement apprécié. Il s'agit d'un cahier de bord humoristique, ubuesque et satirique tenu par les étudiants de la classe préparatoire de khâgne du lycée pendant l'année 1967-1968. Ce cahier servait en quelque sorte d'exutoire à une jeunesse qui commençait à s'agiter et se révolter contre l'autorité et le conformisme de l'époque, juste avant les événements de Mai 1968. Les étudiants y exprimaient librement leurs idées contestataires, dans un esprit et un langage propres à cette classe préparatoire. On y trouve des photographies de défilés d'étudiants et de bizutages à la gloire d'Ubu et de son auteur, l'ex-khâgneux Alfred Jarry, des textes et dessins caricaturaux dans l'esprit de la khâgne, des pamphlets sur les personnalités politiques de l'époque, avec notamment un focus sur un illustre hypokhâgneux dulycée Fermat de la 
promotion 1927-1928, Georges Pompidou, Premier ministre du général de Gaulle au moment de la rédaction de ce cahier ${ }^{40}$.

$\mathrm{Au}$ cours de l'intervention des archives départementales dans le lycée Fermat, un professeur d'histoire du lycée en charge d'un projet Patrimoine avec sa classe préparatoire, Chartes a souhaité faire profiter ses étudiants du travail en cours sur les archives de leur lycée et leur faire rencontrer les acteurs professionnels. Une visite des bâtiments des archives départementales a été organisée pour le groupe avec présentation de l'institution, de ses missions, de son rôle auprès des administrations et de ses services dans la préservation des archives publiques. Quelques documents remarquables ont été exposés et leur contexte de production explicité lors de cette visite découverte: des anciennes photographies de classes du lycée, de beaux parchemins, des registres du parlement de Toulouse, des enluminures, des plans anciens, et en particulier, le testament olographe de Pierre de Fermat ${ }^{41}$ (4 mars 1660). Cette coopération avec le corps enseignant du lycée Fermat est appelée à se renouveler et constitue un exemple du rôle culturel et patrimonial des archives départementales.

Cette intervention ciblée des archives départementales a eu un impact positif en matière de communication culturelle et de pratique archivistique au sein de l'établissement scolaire. En effet, la présence régulière d'un archiviste dans les locaux du lycée pendant près de deux ans a permis d'éclairer les services demandeurs de conseils d'archivage et de répondre à leurs questions concernant les méthodes de classement. La décision de poursuivre les transferts annuels des dossiers d'élèves conduira au versement d'autres archives, qui viendront compléter et enrichir ce fonds, notamment les projets pédagogiques conduits par l'établissement: ils apportent un éclairage intéressant, sur le plan local, des politiques ministérielles et académiques.

Ce travail entre les deux entités, confirmé par le souhait des professeurs de faire découvrir les archives départementales à leurs étudiants, est un bel exemple de coopération entre un établissement d'enseignement et un service d'archives. Ceci ne va pas toujours de soi dans d'autres collectivités ou avec d'autres établissements, plus réticents à confier leurs archives auxquelles les professeurs sont attachés ${ }^{42}$, ou qui ne disposent pas de personnel dédié à leur traitement. La collecte s'est ici déroulée au mieux, grâce à la volonté du proviseur et du personnel d'encadrement, tous convaincus de l'intérêt patrimonial des archives.

5 Les archives départementales ont saisi là une occasion précieuse d'apporter leur appui et de mettre leurs compétences professionnelles au service d'un établissement ancien doté d'un très riche patrimoine. Ainsi garantissent-elles la préservation de la mémoire du lycée et la valorisation de son patrimoine archivistique unique. Au-delà de ce cas particulier, elles ont aussi enrichi les fonds lacunaires d'archives d'établissements scolaires, contribuant à la poursuite des recherches sur l'histoire de l'enseignement. 


\section{NOTES}

1. Archives départementales de la Haute-Garonne (ci-après $A D H G$ ), fonds 204J : 0,3 mètre linéaire.

2. Pour en savoir plus sur l'ancien collège des Jésuites de Toulouse, voir https:// www.pop.culture.gouv.fr/notice/merimee/PA31000080 [lien valide en mars 2021].

3. En découvrir plus sur l'hôtel Bernuy via https://www.pop.culture.gouv.fr/notice/merimee/ PA00094533 [lien valide en mars 2021].

4. En savoir plus par la notice disponible en ligne: https://ressourcespatrimoines.laregion.fr/ ark:/46855/inventaire_IM31001842?posInSet=1\&queryId=bb598993-83fb-4b9c-9e37-0554de5fcb1c [lien valide en mars 2021].

5. En savoir plus par la notice disponible en ligne: https://ressourcespatrimoines.laregion.fr/ ark:/46855/inventaire_IM31100078?posInSet=2\&queryId=bb598993-83fb-4b9c-9e37-0554de5fcb1c [lien valide en mars 2021].

6. Circulaire AD 70-5 des archives de l'Éducation nationale disponible en ligne, https:// francearchives.fr/file/00289437faee3824edef7f7ef82ee23d30169016/static_1006.pdf [lien valide en mars 2021].

7. AD HG, versements $2971 \mathrm{~W}$ versé en 1971 (1865-1961) ; $3751 \mathrm{~W}$ versé en 1979 (1889-1968); 5280 W versé en 1983 (1934-1960).

8. Pour plus d'informations, voir : https://francearchives.fr/fr/circulaire/DPACI_RES_2005_003 ; le document est disponible en ligne, https://francearchives.fr/fr/file/ 9c9646718ba80c848f5029724ac465e1b76adea6/static_892.pdf [liens valides en mars 2021].

9. Document disponible en ligne, https://francearchives.fr/fr/file/ a9edc3f5b0b78bbeb308b72ca6b8fd7379032208/static_1061.pdf [lien valide en mars 2021].

10. Archives courantes: documents produits ou reçus dans l'environnement professionnel immédiat, conservés dans le bureau de l'agent qui les produit (le producteur).

Archives intermédiaires : documents consultés occasionnellement, soit en vertu de prescriptions légales, soit comme objets de référence. Ils sont regroupés dans des dossiers clos mais conservés à proximité pour des raisons d'activité, d'une éventuelle réouverture ou des prescriptions légales.

Archives historiques ou définitives: documents à conserver indéfiniment pour leur intérêt administratif, juridique ou historique.

11. Voir https://archives.haute-garonne.fr/n/colleges-et-lycees/n:255 [lien valide en mars 2021]. 12. Lorsqu'un archiviste effectue le traitement d'un fonds d'archives, il se base sur les instructions ministérielles pour sélectionner les documents à classer et conserver et ceux qui sont à détruire.

13. Le sort final d'un document d'archives est l'entrée qui renseigne sur sa destination au terme de la durée d'utilité administrative ou durée de conservation dans le service imposé par les textes réglementaires ; c'est là qu'est précisé si le document doit être conservé, donc versé aux archives départementales (en totalité ou échantillonné) ou détruit après visa des archives départementales.

14. Au titre du contrôle scientifique et technique des archives départementales sur les archives publiques, la destruction d'archives est la procédure réglementée qui consiste à soustraire un dossier ou un ensemble de dossiers du fonds auquel il appartient, car ils sont dépourvus d'utilité administrative et d'intérêt historique. Ces documents sont alors décrits sur un bordereau soumis au visa du directeur des archives départementales pour accord de destruction.

15. Une boîte d'archives classique est une boîte en carton de format A4 («boîte Cauchard ») qui sert à ranger des documents de taille standard. 
16. Un service producteur d'archives est un service dans une administration ou une collectivité constitué d'agents amenés à produire ou à recevoir des documents administratifs dans l'exercice de leur activité professionnelle.

17. Premier versement: 7 décembre 2017 (articles 1-210). Deuxième versement: 3 mai 2018 (articles 211-336). Troisième versement : 22 juin2018 (articles 337-414). Quatrième versement : 27 mai2019 (articles 415-446). Cinquième versement : 25 juin2019 (articles 447-502). Janvier 2020 : nouveau versement de dossiers d'élèves (articles 503-535).

18. Voir https://archives.haute-garonne.fr/archives/archives/fonds/FRAD031_lycees/ view:fonds/n:3 [lien valide en mars 2021].

19. Consulter le document via https://francearchives.fr/circulaire/CADRE_METHODO_2014_07 [lien valide en mars 2021].

20. Un article d'archives est un ensemble de pièces de même provenance, se rapportant à un même objet ou à une même affaire et dont l'importance matérielle n'excède pas la capacité d'une unité matérielle de conditionnement. L'article constitue tout à la fois une unité intellectuelle de description et l'unité matérielle pour la cotation, le rangement et la communication des documents d'archives.

21. AD HG, versement $8090 \mathrm{~W}$.

22. AD HG, 8090 W 86-300; 415-446; 472-535.

23. AD HG, 8090 W 9-16; 308.

24. AD HG, versements $2971 \mathrm{~W} ; 3751 \mathrm{~W}$.

25. AD HG, 8090 W 357-358.

26. AD HG, 8090 W 347.

27. AD HG, 8090 W 32 ; 34 ; 302-304 ; 387 ; 410-412.

28. AD HG, 8090 W 360 .

29. AD HG, 8090 W 359.

30. AD HG, 8090 W 447-448.

31. AD HG, 8090 W 362.

32. AD HG, 8090 W 363,365.

33. AD HG, 8090 W 325-331.

34. AD HG, 8090 W 361.

35. AD HG, 8090 W 48-85 ; 332-337.

36. AD HG, 8090 W 307, 332.

37. AD HG, 8090 W 40-43.

38. AD HG, 8090 W 138.

39. AD HG, 8090 W 308.

40. AD HG, 8090 W 359001.

41. AD HG, Rés. 48 (3 E 11835, pièce 3725).

42. Certains professeurs d'histoire peuvent avoir besoin de s'appuyer sur des documents d'archives pour illustrer leurs cours ; les archives servent parfois de support pédagogique.

\section{RÉSUMÉS}

La collecte des archives du lycée Pierre-de-Fermat à Toulouse a été organisée pendant les travaux de restructuration des bâtiments du lycée, durant lesquels la conservation des archives a dû être 
externalisée, en attendant leur installation dans de nouveaux locaux. Les archives départementales de la Haute-Garonne sont intervenues à partir de 2017, lors du rapatriement des archives dans les nouveaux locaux du lycée, pour effectuer sur place, durant presque deux ans, des opérations de tri, de classement, d'éliminations réglementaires et de versement d'archives définitives aux archives départementales. Auterme de ce traitement d'arriérés, un fonds d'archives de plus de 50 mètres linéaires est venu enrichir le fonds du lycée Pierre-de-Fermat déjà conservé aux archives départementales. Le versement est composé de documents relatifs à la vie de l'établissement entre 1855 et 2011. On y trouve notamment les documents produits à l'occasion du bicentenaire du lycée (1806-2006), une collection de photographies de classes de 1903 à 2011, ainsi que des documents concernant des personnages illustres ayant fréquenté cet établissement (Georges Canguilhem, Georges Pompidou, Bernard Maris).

Cette opération de collecte est remarquable, dans le sens où le lycée Pierre-de Fermat est l'un des établissements d'enseignement public les plus anciens et les plus prestigieux de Toulouse. En effet, le lycée descend en droite ligne et presque sans rupture de continuité du collège des Jésuites autorisé par édit d'Henri II, promulgué à Nantes en 1551 et confirmé le 19 décembre 1566. Le lycée a drainé l'élite intellectuelle de la région et n'a cessé d'apporter une contribution importante à la formation des cadres supérieurs de la nation par la création, sous le règne de Louis-Philippe, de classes supérieures destinées à préparer les candidats aux grandes écoles.

The lycée Pierre-de-Fermat's archives were collected in Toulouse following restructuring works in the high school buildings, during which the conservation of the archives had to be outsourced before they were moved to new premises. The archives départementales de la Haute-Garonne (the "Regional Archives of Haute-Garonne") intervened from 2017, during the repatriation of the archives to the new high school premises, to carry out on site, for almost two years, operations of sorting, classification, regulatory eliminations and to transfer the remaining archives to the Regional Archives.

At the end of these operations, more than 50 linear meters of documents were added to the lycée Pierre-de-Fermat's archival fonds that were already preserved in the Regional Archives. The transfer consists of documents relating to the life of the school between 1855 and 2011. Particularly notable are the documents produced on the occasion of the high school bicentenary (1806-2006), a collection of class pictures from 1903 to 2011, as well as documents regarding illustrious figures who attended the institution (Georges Canguilhem, Georges Pompidou, Bernard Maris).

This collection operation was remarkable, in the sense that the lycée Pierre-de-Fermat is one of the oldest and most prestigious public education institutions in Toulouse.

Indeed, the high school is directly, and with almost no breach in continuity, descended from the Collège des Jésuites, authorised by edict of King Henri II of France, promulgated in Nantes in 1551 and confirmed on 19 December 1566. The high school has appealed to the local intellectual elite and has constantly made significant contributions to the training of senior public administration managers, since the creation, during the reign of Louis-Philippe, of upper grades intended to prepare candidates for the "Grandes écoles", French prestigious higher education institutions.

\section{INDEX}

Keywords : lycée Pierre-de-Fermat in Toulouse, high school, secondary school, archives, education, pupil, student, professor, departmental archives, regional archives

Mots-clés : lycée Pierre-de-Fermat de Toulouse, lycée, archives, enseignement, élève, étudiant, professeur, archives départementales 
AUTEUR

\section{MARILYS SANCHIS}

Secrétaire de documentation, archives départementales de la Haute-Garonne (Toulouse)

marilys.sanchis@cd31.fr 